\title{
Stress-induced phosphorylation of SNAP-25
}

Saori Yamamori ${ }^{\mathrm{a},{ }^{*}}$, Daichi Sugaya ${ }^{\mathrm{a}}$, Yuuki Iida ${ }^{\mathrm{b}}$, Hirotoshi Kokubo ${ }^{\mathrm{a}}$, Makoto Itakura ${ }^{\mathrm{a}}$, Eiji Suzuki $^{\mathrm{c}}$, Masakazu Kataoka ${ }^{\mathrm{d}}$, Hitoshi Miyaoka ${ }^{\mathrm{b}}$, and Masami Takahashi ${ }^{\mathrm{a}}$

a'Department of Biochemistry, Kitasato University School of Medicine, Sagamihara, Kanagawa 252-0374, Japan

${ }^{\mathrm{b}}$ Department of Psychiatry, Kitasato University School of Medicine, Sagamihara, Kanagawa 252-0380, Japan

'International University of Health and Welfare Atami Hospital, Atami, Shizuoka 413-0012, Japan ${ }^{\mathrm{d}}$ Department of Environmental Science and Technology, Faculty of Engineering, Shinshu University, Nagano-shi, Nagano, 380-8553, Japan

${ }^{*}$ Corresponding author:

Saori Yamamori

Tel: +81-42-778-8626

E-mail: yamamori@med.kitasato-u.ac.jp

Abbreviations:

HPA-axis: hypothalamic-pituitary-adrenal-axis

SNAP-25: synaptosomal-associated protein of $25 \mathrm{kDa}$ 


\begin{abstract}
Synaptosomal-associated protein of $25 \mathrm{kDa}$ (SNAP-25), a t-SNARE protein, plays a crucial role in neurotransmitter release by exocytosis. Protein kinase C phosphorylates SNAP-25 at Ser ${ }^{187}$, however the physiological significance of this phosphorylation event in brain function remains unclear. In the present study, we found that SNAP-25 phosphorylation increased rapidly in the mouse brain following cold-water restraint stress. Both basal and stress-induced phosphorylation of SNAP-25 were high in stress-related brain regions, including the cerebral cortex, hippocampus, and amygdala, and the extent of phosphorylation increased with increasing amounts of stress. Intravenous administration of adrenaline increased SNAP-25 phosphorylation, although stress-induced phosphorylation was still observed in adrenalectomized mice. These results indicate that SNAP-25 phosphorylation is regulated in a stress-dependent manner through both central and peripheral mechanisms.
\end{abstract}

Key words: Protein kinase C, SNARE protein, exocytosis, presynaptic regulation 


\section{Introduction}

Synaptic transmission involves the release of neurotransmitters from presynaptic terminals by exocytosis. Protein kinases both activate and inhibit neurotransmitter release in various contexts $[25,26]$ and are important regulators of synaptic plasticity underlying higher brain functions, such as learning and memory. Synaptosomal-associated protein of $25 \mathrm{kDa}$ (SNAP-25), a t-SNARE protein, plays an essential role in neurotransmitter release [18]. In several secretory cell types, Ser ${ }^{187}$ in the C-terminus of SNAP-25 is phosphorylated by protein kinase C (PKC) [5, 12, 22, 24]. Moreover, PKC activation enhances the exocytotic release of hormones in endocrine cells by recruiting secretory vesicles to the plasma membrane [13], or by increasing the size of the readily releasable vesicle pool [12, 24]. Notably, SNAP-25 phosphorylation at Ser $^{187}$ is essential for these effects [12]. SNAP-25 is phosphorylated at $\operatorname{Ser}^{187}$ in brain tissue as well $[3,5,7,27]$, although the involvement of this phosphorylation event in the regulation of neurotransmitter release is controversial $[1,17]$.

Although SNAP-25 phosphorylation is dramatically altered in epileptic brains, its status in healthy animals is unclear. In the present study, we found that SNAP-25 phosphorylation is dynamically regulated by stress in stress-related regions of the mouse brain by at least two different mechanisms.

\section{Materials and methods}

\subsection{Antibodies}

Anti-SNAP-25 C-terminus [13], anti-phospho-Ser ${ }^{187}$-SNAP-25 [5], and anti-phospho-Ser ${ }^{120}$-SNAP-23 [28] antibodies were prepared as previously described.

\subsection{Animals}

All experiments, were performed with 9-13 week-old male C57BL/6N (CLEA, Japan) mice. For further details, see Supplementary methods. 


\subsection{Stress treatments}

Cold-water restraint stress: Mice were placed into 50-ml plastic centrifuge tubes with 8 small holes drilled at the top and bottom of the tubes, and were submerged horizontally in water at $18 \pm 1^{\circ} \mathrm{C}$ up to the level of the clavicle for the indicated durations. To observe recovery from stress, some mice were returned to their home cages.

Restraint stress: Mice were placed into the same plastic tubes of cold-water restraint stress, and were kept at room temperature for 30 minutes.

Tape-immobilization stress: Mice were fixed on a table with adhesive tape in the supine position for 30 minutes.

Cold-water forced swim stress: Mice were forced to swim in a pool $(45 \mathrm{~cm} \mathrm{~W} \times 26 \mathrm{~cm} \mathrm{D}$ $\times 30 \mathrm{~cm} \mathrm{H}$ ) with water added to a height of $15 \mathrm{~cm}$ at indicated temperatures for 30 minutes.

Unstressed control: All control mice were placed in home cages.

\subsection{Sample preparation}

Mice were decapitated and blood was collected, as needed. Brains were quickly removed from the skull and placed in ice-cold phosphate buffered saline, separated into each region, and homogenized in homogenization buffer (0.32 M sucrose, 5 mM Hepes-NaOH, pH 7.4) supplemented with phosphatase inhibitors (Phos-stop, Roch). Homogenates were mixed with SDS sample buffer, boiled at $100^{\circ} \mathrm{C}$ for 10 minutes, and stored at $-80^{\circ} \mathrm{C}$ until use for immunoblotting. Sera were stored at $-80^{\circ} \mathrm{C}$ until use.

\subsection{Immunoblotting}

They were performed as previously described [3] (see Supplementary methods). 


\subsection{Statistics}

Multiple comparisons were performed using one-way or two-way analysis of variance (ANOVA) with either Tukey-Kramer's or Dunnett's post hoc correction. $P<0.05$ was considered significant.

\section{Results}

3.1. Cold-water restraint stress induces SNAP-25 phosphorylation in the mouse brain

Mice were subjected to cold-water restraint stress. Serum corticosterone levels increased rapidly and reached a maximal level at about 30 minutes after exposure to stress, confirming the potency of the stress (Fig. 1A). We then examined the extent of $\operatorname{Ser}^{187}$ phosphorylation and total protein levels of SNAP-25 in the hippocampus before and after the stress. Even in stress-free control mice, SNAP-25 phosphorylated at Ser ${ }^{187}$ was detected in hippocampal homogenates, indicating that a fraction of SNAP-25 is phosphorylated in vivo. SNAP-25 phosphorylation increased rapidly in response to stress, with a time-course similar to that of serum corticosterone. However, the amount of total SNAP-25 protein remained constant during the test period. When stressed mice were returned to their home cages, SNAP-25 phosphorylation rapidly decreased and returned to basal levels within 60 minutes (Fig. 1B). Serum corticosterone levels also decreased, but to a lesser extent than was observed with SNAP-25 phosphorylation. These results indicate that SNAP-25 phosphorylation is dynamically regulated in vivo.

SNAP-23 is a homolog of SNAP-25 that is expressed ubiquitously in various tissues. PKC phosphorylates SNAP-23 at several serine residues, including $\operatorname{Ser}^{120}$ [28]. No significant change was observed in SNAP-23 phosphorylation at $\operatorname{Ser}^{120}$ in response to cold-water restraint stress (data not shown).

\subsection{Regional differences in stress-induced SNAP-25 phosphorylation}


Next, we compared levels of total SNAP-25 protein and SNAP-25 phosphorylation in various brain regions with or without cold-water restraint stress (Fig. 2). Although SNAP-25 protein levels remained equal in all brain regions tested, SNAP-25 phosphorylation was high in brain regions involved in stress response, including the cerebral cortex, hippocampus, and amygdala. Furthermore, while cold-water restraint stress further induced SNAP-25 phosphorylation in the cerebral cortex, hippocampus, and amygdala, no further elevation was observed in the hypothalamus or cerebellum.

\subsection{Extent of SNAP-25 phosphorylation depends on degree of stress}

If SNAP-25 phosphorylation occurs as a step in the stress response, a correlation should exist between the degree of stress and extent of SNAP-25 phosphorylation. Tape-immobilization stress is a stronger stress stimulus than restraint stress [20]. SNAP-25 phosphorylation was not significantly increased by acute restraint stress in a small tube, whereas a marked increase was observed after tape-immobilization stress (Fig. 3A). In the cold-water forced swim test, the strength of stress increases with decreasing water temperature. SNAP-25 phosphorylation increased in mice forced to swim as the water temperature was decreased (Fig. 3B). These results collectively show a correlation between the extent of SNAP-25 phosphorylation and degree of stress.

\subsection{Central and peripheral regulation of SNAP-25 phosphorylation in the brain}

Adrenaline is released from the adrenal medulla in response to various stress stimuli. Intraperitoneal administration of adrenaline markedly induced SNAP-25 phosphorylation at Ser ${ }^{187}$ in various brain regions, indicating that SNAP-25 phosphorylation could be regulated, at least in part, through feedback regulation by adrenaline in peripheral tissues (Fig. 4). Although

peripherally-administrated adrenaline induced SNAP-25 phosphorylation in the brain (Fig. 4), it was possible that cold-water restraint stress induced SNAP-25 phosphorylation via a mechanism that did not involve peripheral feedback. To test this possibility, we examined stress-induced SNAP-25 phosphorylation in adrenalectomized mice. Cold-water restraint stress significantly increased serum corticosterone levels in sham-operated mice, but not in adrenalectomized mice (data not shown). In 
contrast, cold-water restraint stress induced SNAP-25 phosphorylation in both sham-operated mice and adrenalectomized mice (Fig. 5). These results indicate that a direct pathway in the brain, which is not downstream of the peripheral feedback system, links stress to SNAP-25 phosphorylation. We also found that stress-induced SNAP-25 phosphorylation was significantly higher in adrenalectomized mice compared to sham-operated mice only in the hippocampus (two-way repeated measures ANOVA interaction effect between stress and adrenalectomy, $p=0.0044$; main effect of stress, $p<0.0001$; main effect of adrenalectomy, $p=0.015$ ). A small but statistically significance increase of SNAP-25 phosphorylation was observed in cerebellum of adrenalectomized mice. The reason is not clear at the present, however, prolonged effects of sham-operation may affect on the response in cerebellum.

\section{Discussion}

In previous studies, SNAP-25 phosphorylation was induced using artificial techniques including PKC activator-like phorbol esters in cultured cells and electrical stimulation in hippocampal slice cultures. In mice, however, only epileptic seizures have successfully induced SNAP-25 phosphorylation. In the present study, we demonstrate for the first time that SNAP-25 phosphorylation is increased in healthy animals subjected to physical stress. This phosphorylation event was specific to SNAP-25, as SNAP-23, a ubiquitously expressed SNAP-25 homolog, was not phosphorylated under the same conditions. Basal and stress-induced SNAP-25 phosphorylation was high in brain regions suggested to be involved in stress response, and a correlation was found between the extent of SNAP-25 phosphorylation and degree of stress. Collectively, these results suggest that SNAP-25 phosphorylation plays an important role in stress response in the brain.

When the brain senses stress, the HPA-axis and sympathetic nervous system are activated, leading to glucocorticoid and adrenaline release from the adrenal glands into the blood. The released glucocorticoid and adrenaline affect not only peripheral cells, but also the brain by feedback mechanisms. This suggests that there are two possible pathways for regulating stress-induced SNAP-25 phosphorylation in the brain-one is direct (i.e., without participation of peripheral 
mechanisms) and the other is a feedback mechanism from the peripheral system. Our results support both mechanisms. For example, peripherally-administered adrenaline increased SNAP-25 phosphorylation in the brain. Since adrenaline does not cross the blood-brain barrier, adrenaline likely exerted its effect on the brain by activating the vagus nerve [11]. On the other hand, adrenalectomized mice also exhibited stress-induced SNAP-25 phosphorylation, which would suggest that peripheral feedback is not essential for SNAP-25 phosphorylation. While we found an interaction effect between stress and adrenalectomy in the hippocampus, no such effect was found in the amygdala. The hippocampus and amygdala play reciprocal roles in stress response; the former plays an inhibitory role, while the latter plays an excitatory role. Thus, the interaction effect between stress and adrenalectomy in the hippocampus might be attributable to the characteristic properties of the hippocampus in stress response. However, further studies will be needed to elucidate the functional roles of SNAP-25 phosphorylation in stress response in the brain.

We previously showed that serotonin and dopamine release are markedly reduced in the amygdala of Snap-25 $5^{\text {S187A/S187A }}$ mutant mice [8]. In secretory cells, the activation of PKC by phorbol ester treatment induces SNAP-25 phosphorylation at Ser $^{187}$ and enhances catecholamine and peptide hormone release from these cells [5, 12, 22, 24]. However, in hippocampal glutamatergic neurons, SNAP-25 phosphorylation was dispensable for phorbol ester-induced enhancement of synaptic transmission $[1,17]$. Taken together, these results suggest that SNAP-25 phosphorylation is selectively involved in the regulation of monoamine release in the mouse brain. Various stressors induce monoamine release in specific brain regions $[4,16,29]$. Thus, it is possible that SNAP-25 phosphorylation participates in the stress response by regulating monoamine release in the brain. SNAP-25 is a multifunctional protein that plays a role in several processes unrelated to neurotransmitter release, such as neurite extension [14], incorporation of ion channels and receptors into the plasma membrane by exocytosis $[9,10,15,21]$, and regulation of ion channel function $[2,6$, 19]. At least some of these processes are considered to be regulated by SNAP-25 phosphorylation at 
$\operatorname{Ser}^{187}[9,10,15,21]$. This raises the possibility that SNAP-25 phosphorylation may modulate the function of stress-related neurons via mechanisms independent of monoamine release. Further studies will be necessary to address these possibilities.

\section{Acknowledgements}

This work was supported by: Grants-in-Aid 18300127 and 21300141 for Scientific Research (B), 24700385 for Young Scientists (B), and 21650091 for Challenging Exploratory Research from the Japan Society for the Promotion of Science, and a Grant-in-Aid for Core Research for Evolutional Science and Technology from the Japan Science and Technology Agency.

\section{Appendix A. Supplementary methods}

Supplementary methods associated with this article can be found, in the online version, at http://----. 


\section{References}

[1] M.F. Finley, R.H. Scheller, D.V. Madison, SNAP-25 Ser ${ }^{187}$ does not mediate phorbol ester enhancement of hippocampal synaptic transmission, Neuropharmacology 45 (2003) 857-862.

[2] Y. He, C.L. Elias, Y.C. Huang, X. Gao, Y.M. Leung, Y. Kang, H. Xie, J.A. Chaddock, R.G. Tsushima, H.Y. Gaisano, Botulinum neurotoxin A and neurotoxin E cleavage products of synaptosome-associated protein of $25 \mathrm{kd}$ exhibit distinct actions on pancreatic islet beta-cell Kv2.1 channel gating, Pancreas 36 (2008) 10-17.

[3] Y. Iida, S. Yamamori, M. Itakura, H. Miyaoka, M. Takahashi, Protein phosphatase 2A dephosphorylates SNAP-25 through two distinct mechanisms in mouse brain synaptosomes, Neurosci. Res. 75 (2013) 184-189.

[4] A. Imperato, S. Puglisi-Allegra, P. Casolini, L. Angelucci, Changes in brain dopamine and acetylcholine release during and following stress are independent of the pituitary-adrenocortical axis, Brain Res. 538 (1991) 111-117.

[5] S. Iwasaki, M. Kataoka, M. Sekiguchi, Y. Shimazaki, K. Sato, M. Takahashi, Two distinct mechanisms underlie the stimulation of neurotransmitter release by phorbol esters in clonal rat pheochromocytoma PC12 cells, J. Biol. Chem. 128 (2000) 407-414.

[6] S.E. Jarvis, G.W. Zamponi, Interactions between presynaptic $\mathrm{Ca}^{2+}$ channels, cytoplasmic messengers and proteins of the synaptic vesicle release complex, Trends. Pharmacol. Sci. 22 (2001) 519-525.

[7] M. Kataoka, R. Kuwahara, R. Matsuo, M. Sekiguchi, K. Inokuchi, M. Takahashi, Developmentand activity-dependent regulation of SNAP-25 phosphorylation in rat brain, Neurosci. Lett. 407 (2006) 258-262.

[8] M. Kataoka, S. Yamamori, E. Suzuki, S. Watanabe, T. Sato, H. Miyaoka, S. Azuma, S. Ikegami, R. Kuwahara, R. Suzuki-Migishima, Y. Nakahara, I. Nihonmatsu, K. Inokuchi, Y. Katoh-Fukui, M. Yokoyama, M.Takahashi, A single amino acid mutation in SNAP-25 induces 
anxiety-related behavior in mouse, PLoS one 6 (2011) e25158.

[9] J.Y. Lan, V.A. Skeberdis, T. Jover, S.Y. Grooms, Y. Lin, R.C. Araneda, X. Zheng, M.V. Bennett, R.S. Zukin, Protein kinase C modulates NMDA receptor trafficking and gating, Nat. Neurosci. 4 (2001) 382-390.

[10] C.G. Lau, Y. Takayasu, A. Rodenas-Ruano, A.V. Paternain, J. Lerma, M.V. Bennett, R.S.Zukin, SNAP-25 is a target of protein kinase C phosphorylation critical to NMDA receptor trafficking, J. Neurosci. 30 (2010) 242-254.

[11] J.L. Mcgaugh, L. Cahill, B. Roozendaal, Involvement of the amygdala in memory storage: Interaction with other brain systems, Proc. Natl. Acad. Sci. U.S.A. 93 (1996) 13508-13514.

[12] G. Nagy, U. Matti, R.B. Nehring, T. Binz, J. Rettig, E. Neher, J.B. Sorensen, Protein kinase C-dependent phosphorylation of synaptosome-associated protein of $25 \mathrm{kDa}$ at $\mathrm{Ser}^{187}$ potentiates vesicle recruitment, J. Neurosci. 22 (2002) 9278-9786.

[13] C. Oho, S. Seini, M. Takahashi, Expression and complex formation of soluble N-ethyl-maleimide-sensitive facor attachment protein (SNAP) receptors in clonal rat endcrine cells, Neurosci. Lett. 186 (1995) 208-210.

[14] A. Osen-Sand, M. Catsicas, J.K. Staple, K.A. Jones, G. Ayala, J. Knowles, G. Grenningloh, S. Catsicas, Inhibition of axonal growth by SNAP-25 antisense oligonucleotides in vitro and in vivo, Nature 364 (1993) 445-448.

[15] D. Pozzi, S. Condliffe, Y. Bozzi, M. Chikhladze, C. Grumelli, V. Proux-Gillardeaux, M .Takahashi, S. Franceschetti, C. Verderio, M. Matteoli, Activity-dependent phosphorylation of Ser $^{187}$ is required for SNAP-25-negative modulation of neuronal voltage-gated calcium channels, Proc. Natl. Acad. Sci. U.S.A. 105 (2008) 323-328.

[16] S. Puglisi-Allegra, A. Imperato, L. Angelucci, S. Cabib, Acute stress induces time-dependent responses in dopamine mesolimbic system, Brain Res. 554 (1991) 217-222.

[17] J.S. Rhee, A. Betz, S. Pyott, K. Reim, F. Varoqueaux, I. Augustin, D. Hesse, T.C. Sudhof, M. 
Takahashi, C. Rosenmund, N. Brose, Phorbol ester- and diacylglycerol-induced augmentation of transmitter release is mediated by Munc13s and not by PKCs, Cell 108 (2002) 121-133.

[18] J. Rizo, T.C. Südhof, The membrane fusion enigma: SNAREs, Sec1/Munc18 proteins, and their accomplices - Guilty as charges?, Annu. Rev. Cell Dev. Biol. 28 (2012) 279-308.

[19] J. Rettinger, Characteristics of $\mathrm{Na}^{+} / \mathrm{K}^{+}$-ATPase mediated proton current in $\mathrm{Na}^{+}$- and $\mathrm{K}^{+}$-free extracellular solutions. Indications for kinetic similarities between $\mathrm{H}^{+} / \mathrm{K}^{+}$-ATPase and $\mathrm{Na}^{+} / \mathrm{K}^{+}$-ATPase, Biochim. Biophys. Acta 1282 (1996) 207-215.

[20] O. Sanchez, A. Arnau, M. Pareja, E. Poch, I. Ramirez, M. Soley, Acute stress-induced tissue injury in mice: differences between emotional and social stress, Cell Stress Chaperones 7 (2002) 36-46.

[21] S. Selak, A.V. Paternain, I.M. Aller, E. Pico', R. Rivera, J. Lerma., Role for SNAP25 in internalization of kainate receptors and synaptic plasticity, Neuron 63 (2009) 357-371.

[22] Y. Shimazaki, T. Nishiki, A. Omori, M. Sekiguchi, Y. Kamata, S. Kozaki, M.Takahashi, Phosphorylation of 25-kDa synaptosome-associated protein, J. Biol. Chem. 271 (1996) $14548-14553$.

[23] Y. Shoji-Kasai, M. Itakura, M. Kataoka, S. Yamamori, M. Takahashi, Protein kinase C-mediated translocation of secretory vesicles to plasma membrane and enhancement of neurotransmitter release from PC12 cells, Eur. J. Neurosci. 15 (2002) 1390-1394.

[24] Y. Shu, X. Liu, Y. Yang, M. Takahashi, K.D. Gillis, Phosphorylation of SNAP-25 at Ser ${ }^{187}$ mediates enhancement of exocytosis by a phorbol ester in INS-1 cells, J. Neurosci. 28 (2008) 21-30.

[25] M. Takahashi, M. Itakura, M. Kataoka , New Aspects of Neurotransmitter Release and Exocytosis: Regulation of Neurotransmitter Release by Phosphorylation, J. Pharmachol. Sci. 93 (2003) 41-45.

[26] K.M.Turner, R.D. Burgoyne, A. Morgan, Protein phosphorylation and the regulation of synaptic 
membrane traffic, Trends in Neurosci. 22 (1999) 459-464.

[27] N.J. Xu, Y.X. Yu, J.M. Zhu, H. Liu, L. Shen, R. Zeng, X. Zhang, G. Pei, Inhibition of SNAP-25 phosphorylation at $\operatorname{Ser}^{187}$ is involved in chronic morphine-induced down-regulation of SNARE complex formation, J. Biol. Chem. 279 (2004) 40601-40608

[28] K. Yasuda, M. Itakura, K. Aoyagi, T. Sugaya, E. Nagata, H. Ihara, M. Takahashi, PKC-dependent inhibition of $\mathrm{Ca}^{2+}$-dependent exocytosis from astrocytes, Glia 59 (2011) 143-151.

[29] A.M. Young, Intracerebral microdialysis in the study of physiology and behaviour, Rev. Neurosci. 4 (1993) 373-395. 


\section{FIGURE LEGENDS}

Fig. 1. Stress-induced phosphorylation of SNAP-25 in the hippocampus and changes in serum corticosterone levels after subjecting mice to cold-water restraint stress.

$\boldsymbol{A}$, Mice were subjected to cold-water restraint stress for the indicated durations. Protein amounts and phosphorylation levels of SNAP-25 in the hippocampus were determined by immunoblotting with anti-SNAP-25 antibody and anti-phospho-Ser ${ }^{187}$-SNAP-25 antibody, respectively. Serum corticosterone levels were determined by ELISA. (Upper) Typical immunoblots. (Lower) Quantification of SNAP-25 phosphorylation (circles) and serum corticosterone levels (triangles) before (open symbols) and after (closed symbols) stress. SNAP-25 phosphorylation was quantified densitometrically by dividing the band intensity value for SNAP-25 phosphorylation by the value for total SNAP-25 protein. Values are expressed relative to that of control mice. $\boldsymbol{B}$, Mice were subjected to cold-water restraint stress for 30 minutes and then returned to their home cages. Protein amounts, phosphorylation levels of SNAP-25 in the hippocampus, and serum corticosterone levels were determined and were represented as in $\boldsymbol{A}$. Values represent mean $\pm \mathrm{SE}$ (A, control, $\mathrm{n}=4$; others, $\mathrm{n}=$ 3; B, 90min, $\mathrm{n}=4$; others, $\mathrm{n}=3$ ). ${ }^{* *}, p<0.01$ and ${ }^{*}, p<0.05$ comparing with and without stress, ${ }^{\#}$, $p<0.01$ and $^{\#}, p<0.05$ comparing before and after returning mice to their home cages, by one-way ANOVA with Tukey-Kramer's post hoc correction.

Fig. 2. Basal and stress-induced SNAP-25 phosphorylation in various brain regions.

Mice were subjected to cold-water restraint stress for 30 minutes. Various brain regions were isolated from either controls (-) or mice subjected to cold-water restraint stress for 30 minutes $(+)$. Protein amounts and phosphorylation levels of SNAP-25 were determined by immunoblotting with anti-SNAP-25 antibody and anti-phospho-Ser ${ }^{187}$-SNAP-25 antibody, respectively. (Upper) Typical immunoblots. Cx, cerebral cortex; HC, hippocampus; Amy, amygdala; HT, hypothalamus; CB, 
cerebellum. (Lower) Quantified results of SNAP-25 phosphorylation with (closed columns) or without (open columns) stress. The values were normalized to those of SNAP-25 amounts in each region, and were expressed as relative values to that in the hippocampus of control mice. The result represents a typical example of three independent experiments. Values represent mean \pm SE $(n=3)$. **, $p<0.01$ comparing with and without stress, by one-way ANOVA with Tukey-Kramer's post hoc correction.

Fig. 3. Extent of SNAP-25 phosphorylation by degree of stress.

$\boldsymbol{A}$, Mice were restrained either in a plastic tube or with adhesive tape for 30 minutes at room temperature. Protein amounts and Phosphorylation levels of SNAP-25 in the hippocampus of either control or stressed mice were determined by immunoblotting with anti-SNAP-25 antibody and anti-phospho-Ser ${ }^{187}$-SNAP-25 antibody, respectively. Values were normalized to those of SNAP-25 amounts and were represented relative to control mice. $\boldsymbol{B}$, Mice were subjected to the forced swim test for 30 minutes at 18,30 or $35 \pm 1^{\circ} \mathrm{C}$, and Protein amounts and Phosphorylation levels of SNAP-25 in the hippocampus were determined and represented as in $\boldsymbol{A}$. Values represent mean \pm SE (n = 3). ${ }^{* *}, p<0.01$ and ${ }^{*}, p<0.05$ compared to control unstressed mice, by one-way ANOVA with Dunnett's post hoc correction.

Fig. 4. Intraperitoneal injection of adrenaline increases SNAP-25 phosphorylation in the mouse brain.

Adrenaline (0.5 mg/kg; ip) was intraperitoneally administered to mice. After 30 minutes, brain regions were isolated from mice receiving either saline (-) or adrenaline (+). Protein amounts and phosphorylation levels of SNAP-25 were determined by immunoblotting with anti-SNAP-25 antibody and anti-phospho-Ser ${ }^{187}$-SNAP-25 antibody, respectively. (Upper) Typical immunoblots. Cx, cerebral cortex; HC, hippocampus; Amy, amygdala; HT, hypothalamus; CB, cerebellum. (Lower) 
Quantified results of SNAP-25 phosphorylation with (closed columns) or without (open columns) administration of adrenaline. Values were normalized to those of SNAP-25 amounts in each region, and were expressed as relative values to saline-injected control mice. Values represent mean \pm SE (n =3). **, $p<0.01$ and *, $p<0.05$ compared to control mouse, by one-way ANOVA with Tukey-Kramer's post hoc correction.

Fig. 5. Basal and stress-induced SNAP-25 phosphorylation in the brain of adrenalectomized mice. Adrenalectomized (ADX) and sham-operated (Sham) mice were restrained in cold water for 30 minutes. Various brain regions were isolated from either control mice (-) or mice subjected to cold-water restraint stress for 30 minutes (+). Protein amounts and phosphorylation levels of SNAP-25 were determined by immunoblotting with anti-SNAP-25 antibody and anti-phospho-Ser ${ }^{187}$-SNAP-25 antibody, respectively. (Upper) Typical immunoblots. Cx, cerebral cortex; HC, hippocampus; Amy, amygdala; HT, hypothalamus; CB, cerebellum. (Lower) Quantified results of SNAP-25 phosphorylation with (closed columns) or without (open columns) stress. Values were normalized to SNAP-25 levels in each region, and were expressed as relative values to that in sham-operated control mice. Values represent mean \pm SE (n = 3 per group). ${ }^{* *}, p<0.01$ and $*, p<$ 0.05 compared to control mice, by two-way ANOVA with Tukey-Kramer's post hoc correction. A significant interaction effect was observed in the hippocampus between stress and adrenalectomy ( $p$ $=0.0044)$. 
A
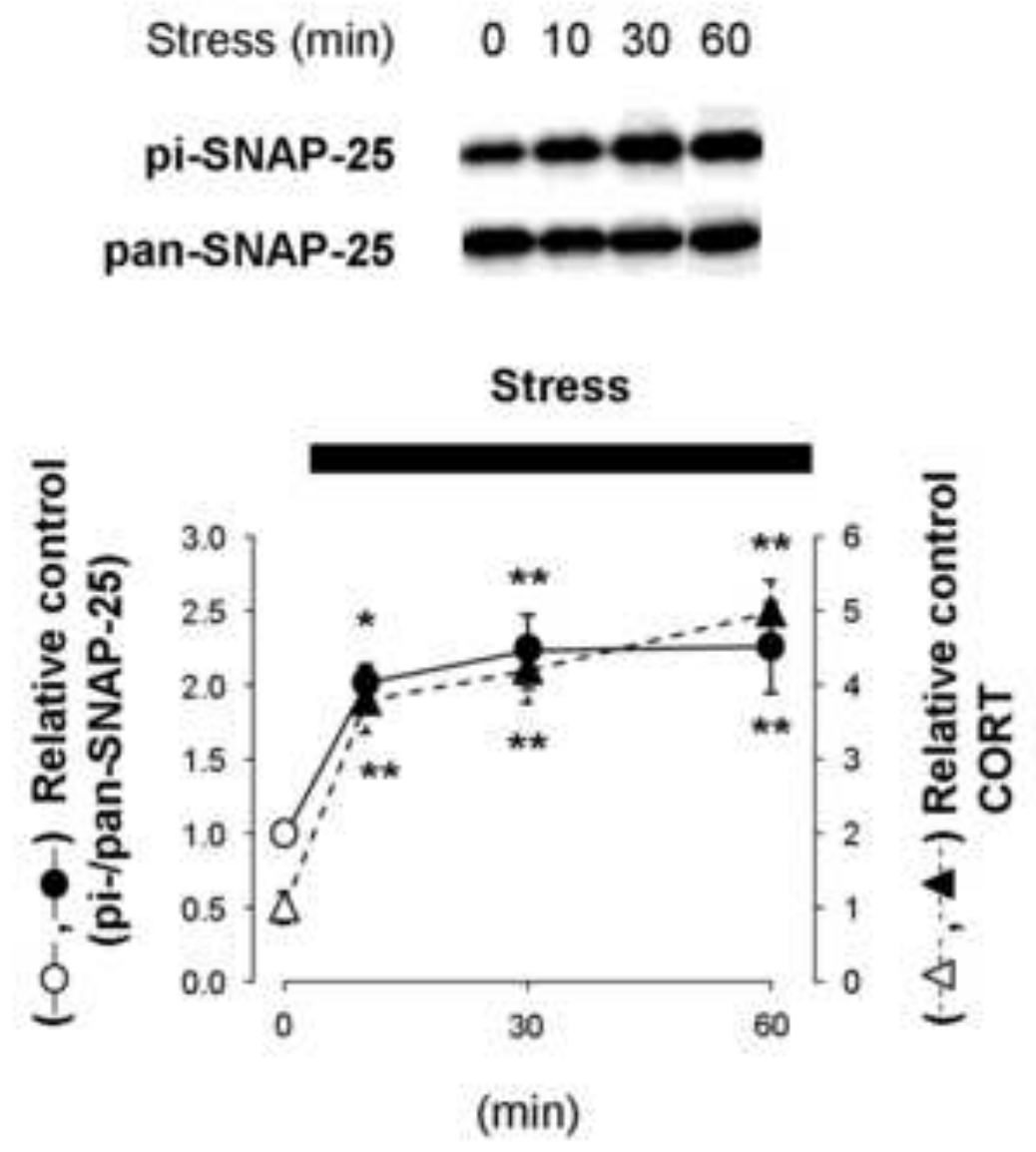

B
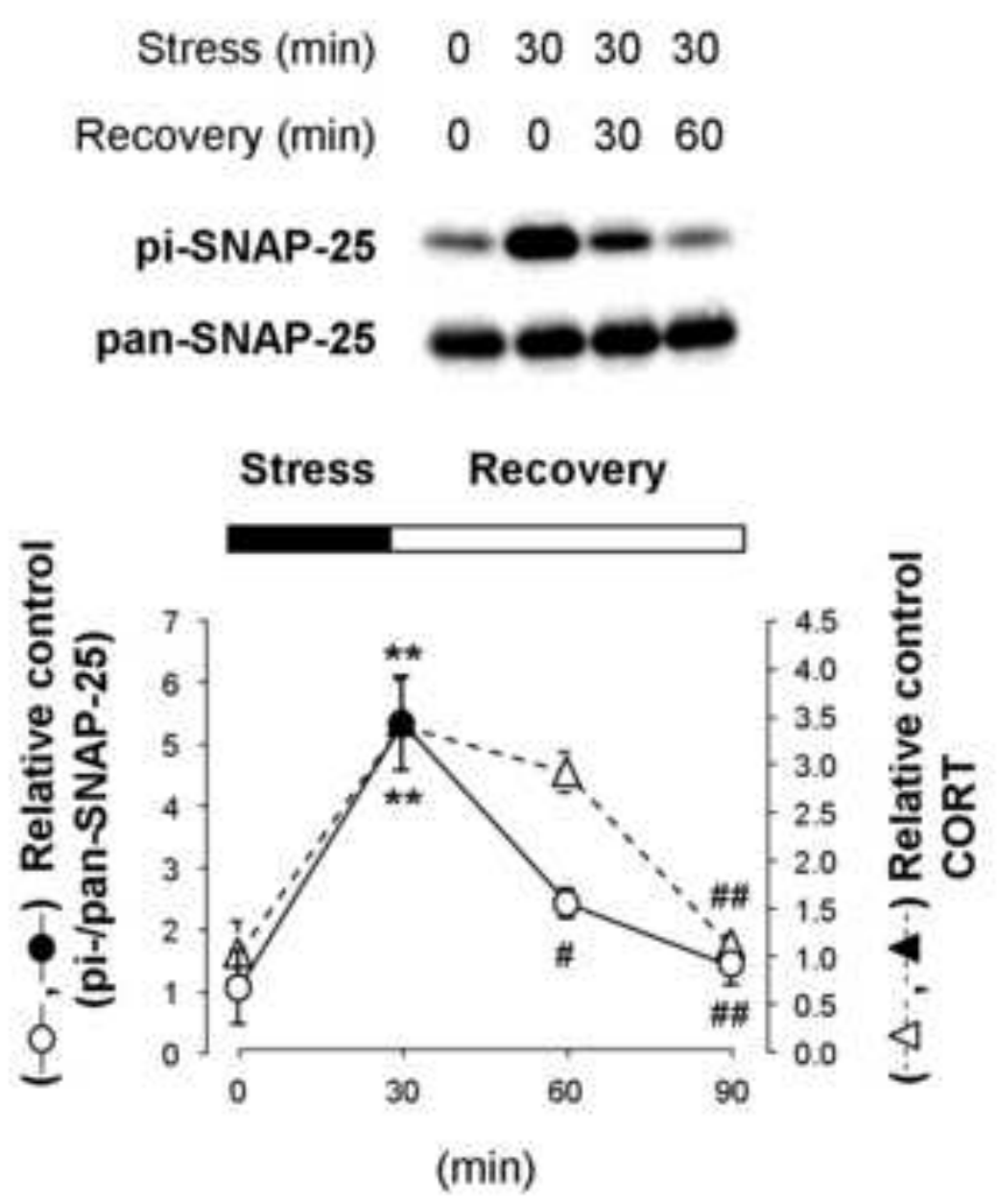


\section{Cx HC Amy HT CB \\ Stress $-+-++_{-}++_{-}+$ \\ Cx HC Amy}

pi-SNAP-25 pan-SNAP-25

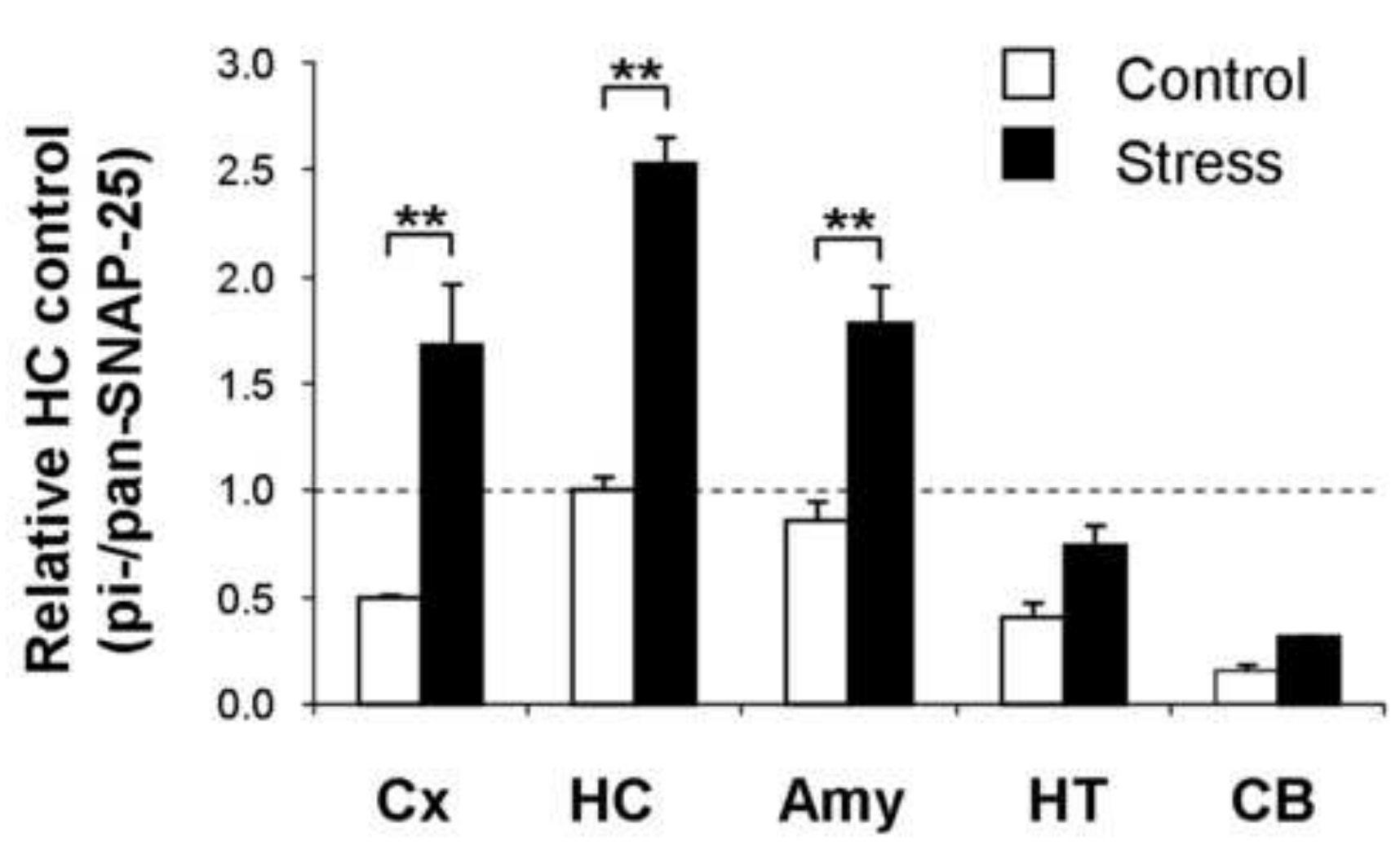

Figure 2

\section{-6-6-0-0}

\section{0}


A
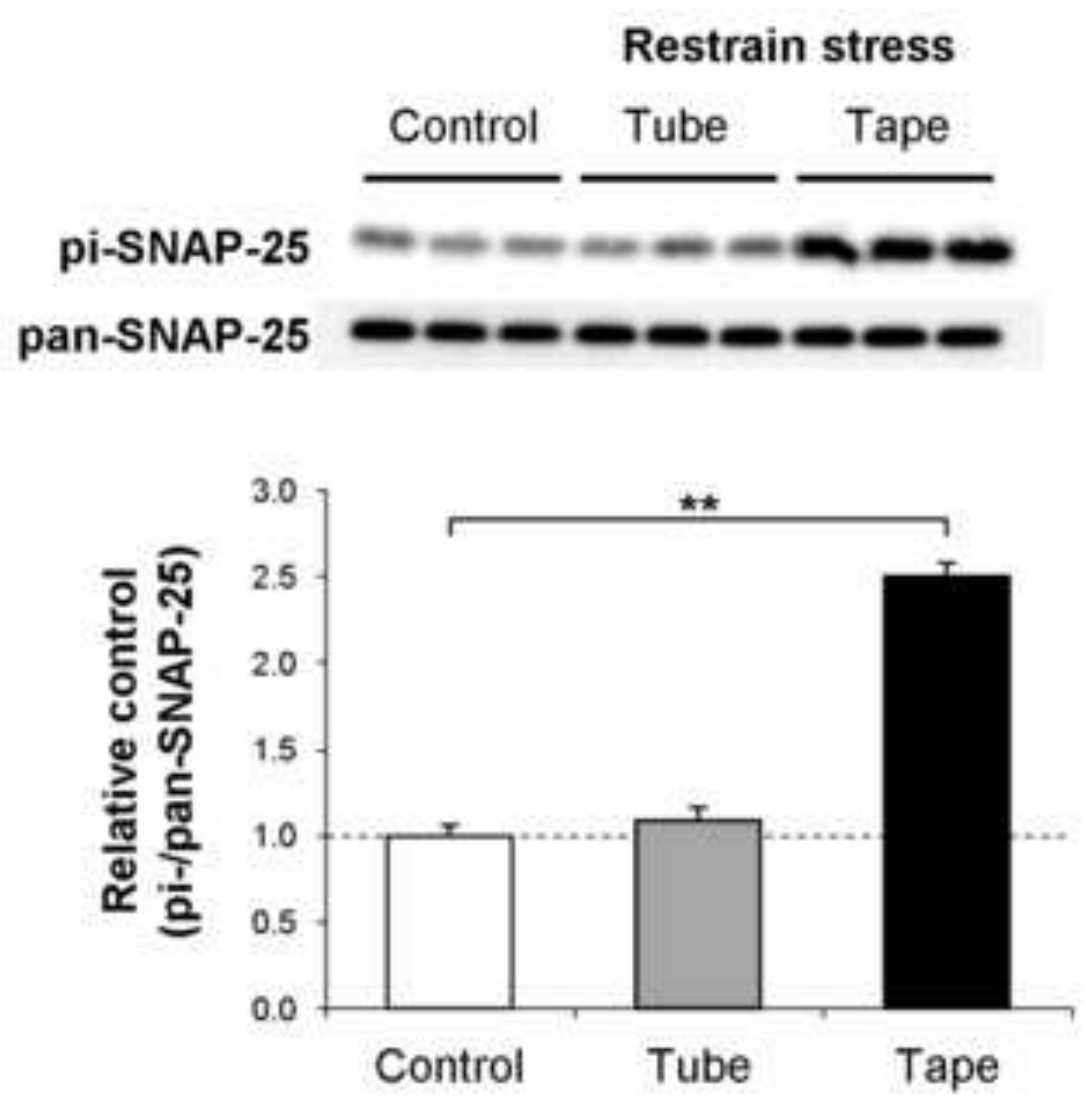

B

Forced swim stress

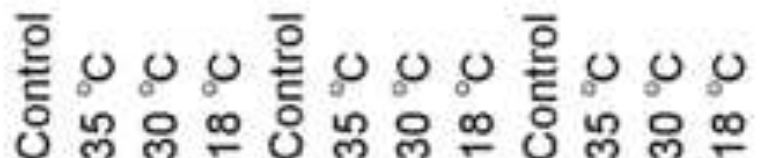

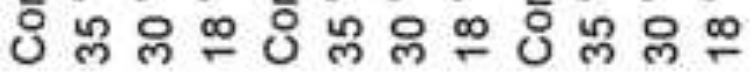
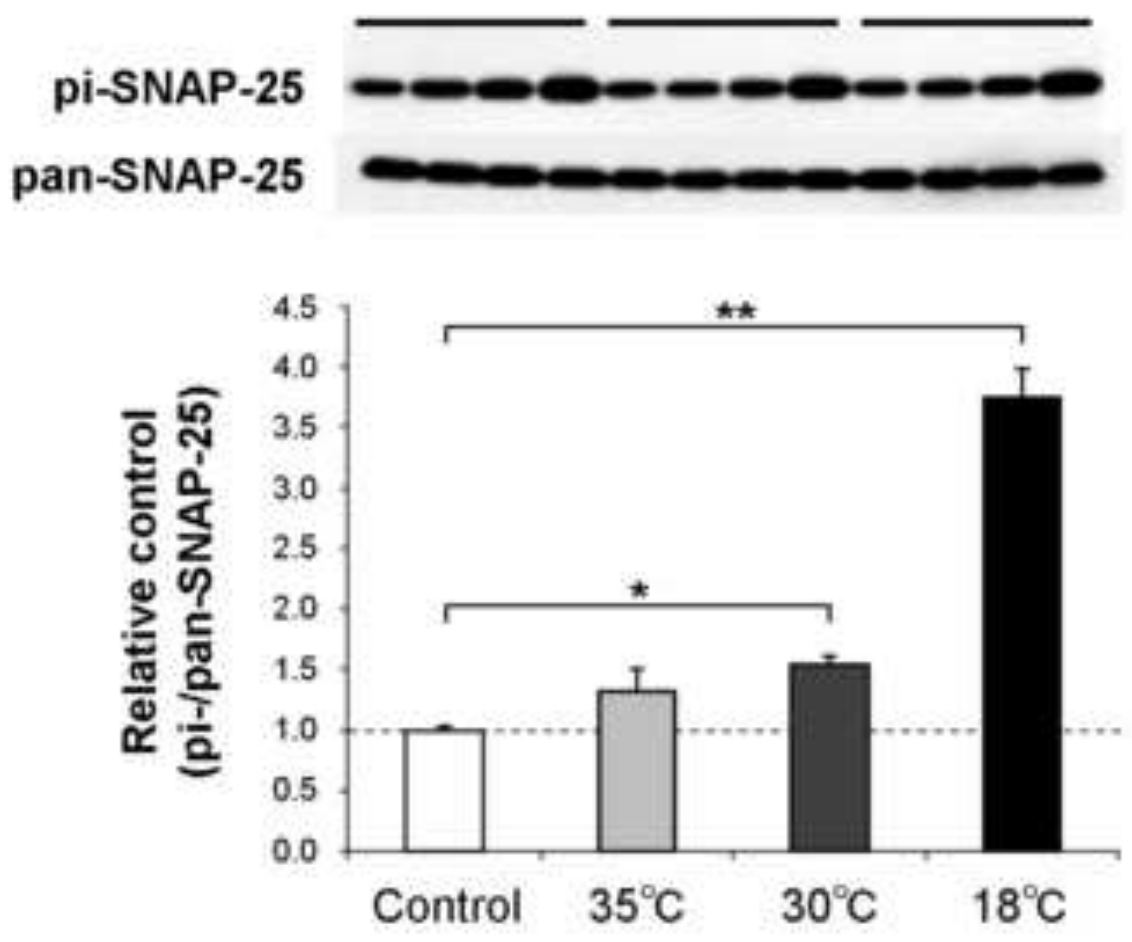


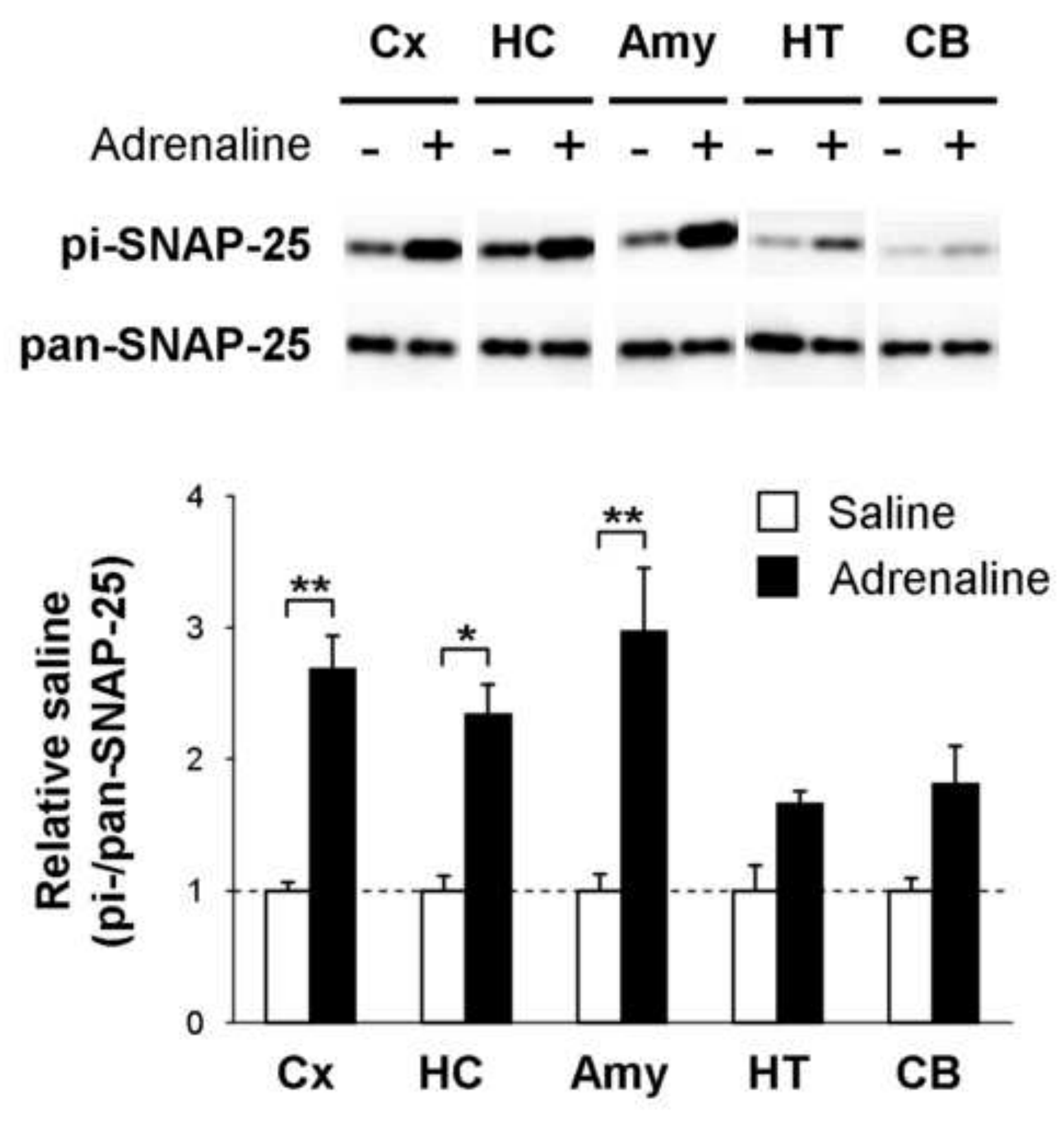
pan-SNAP-25

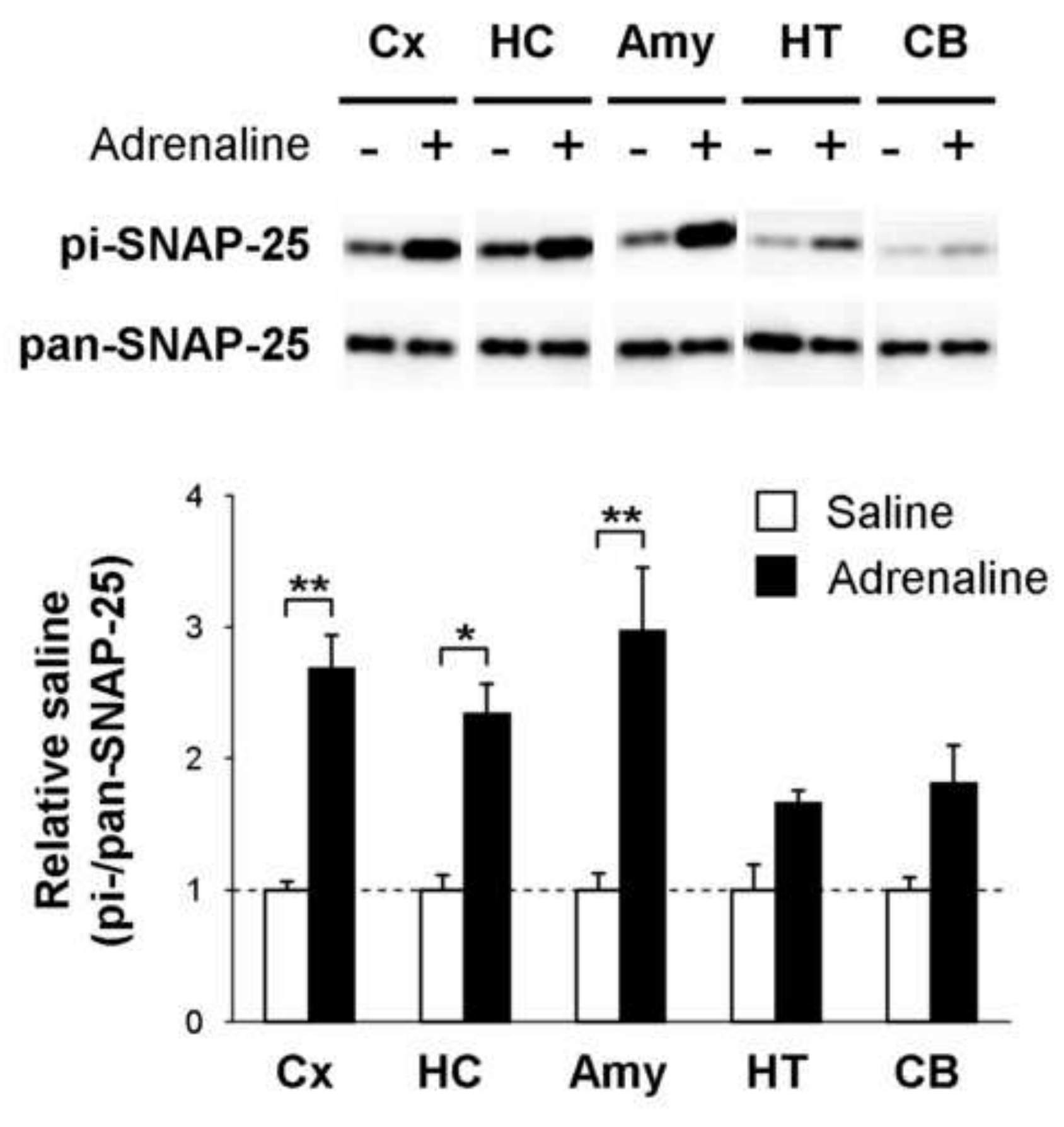

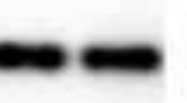

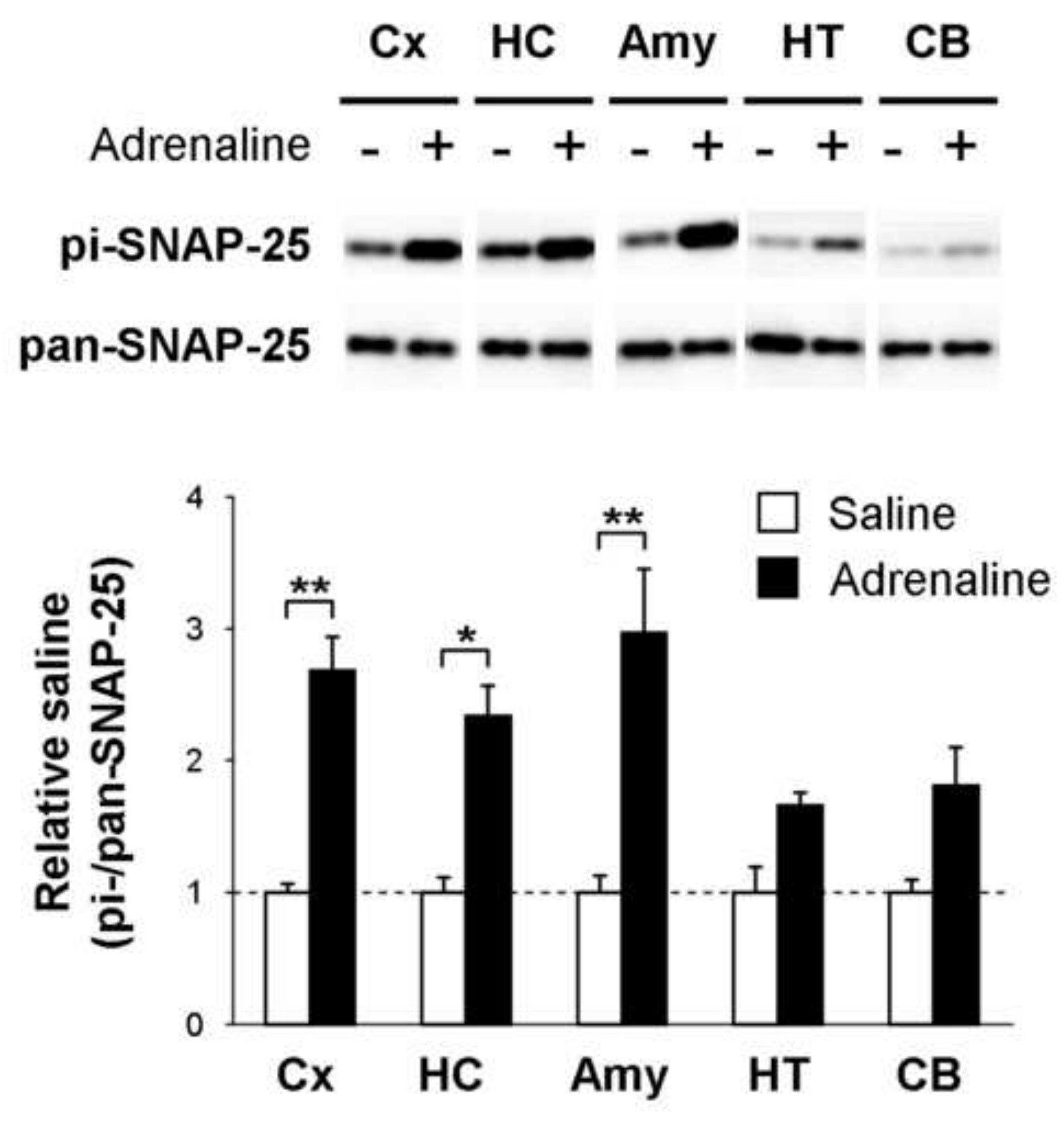

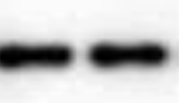
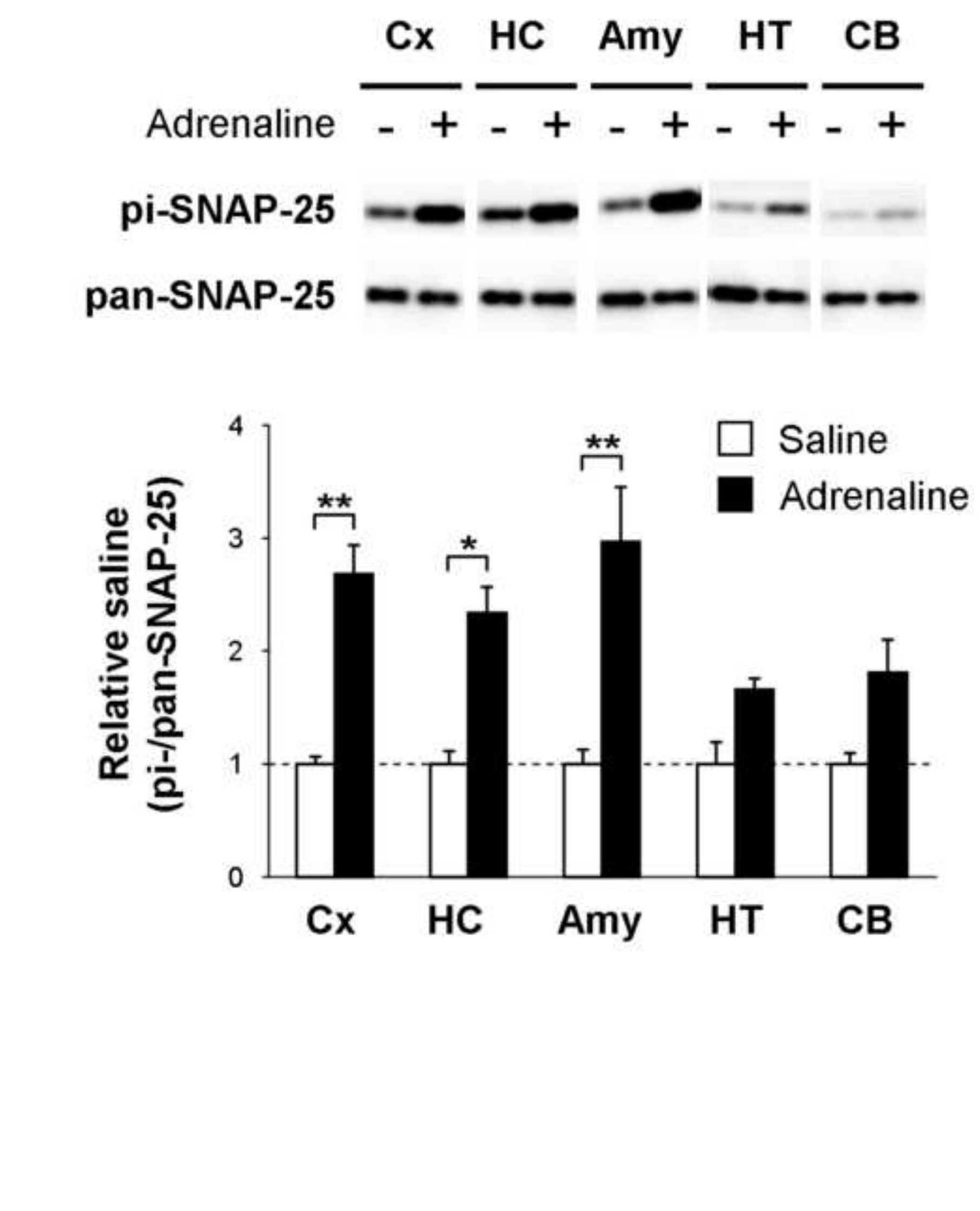

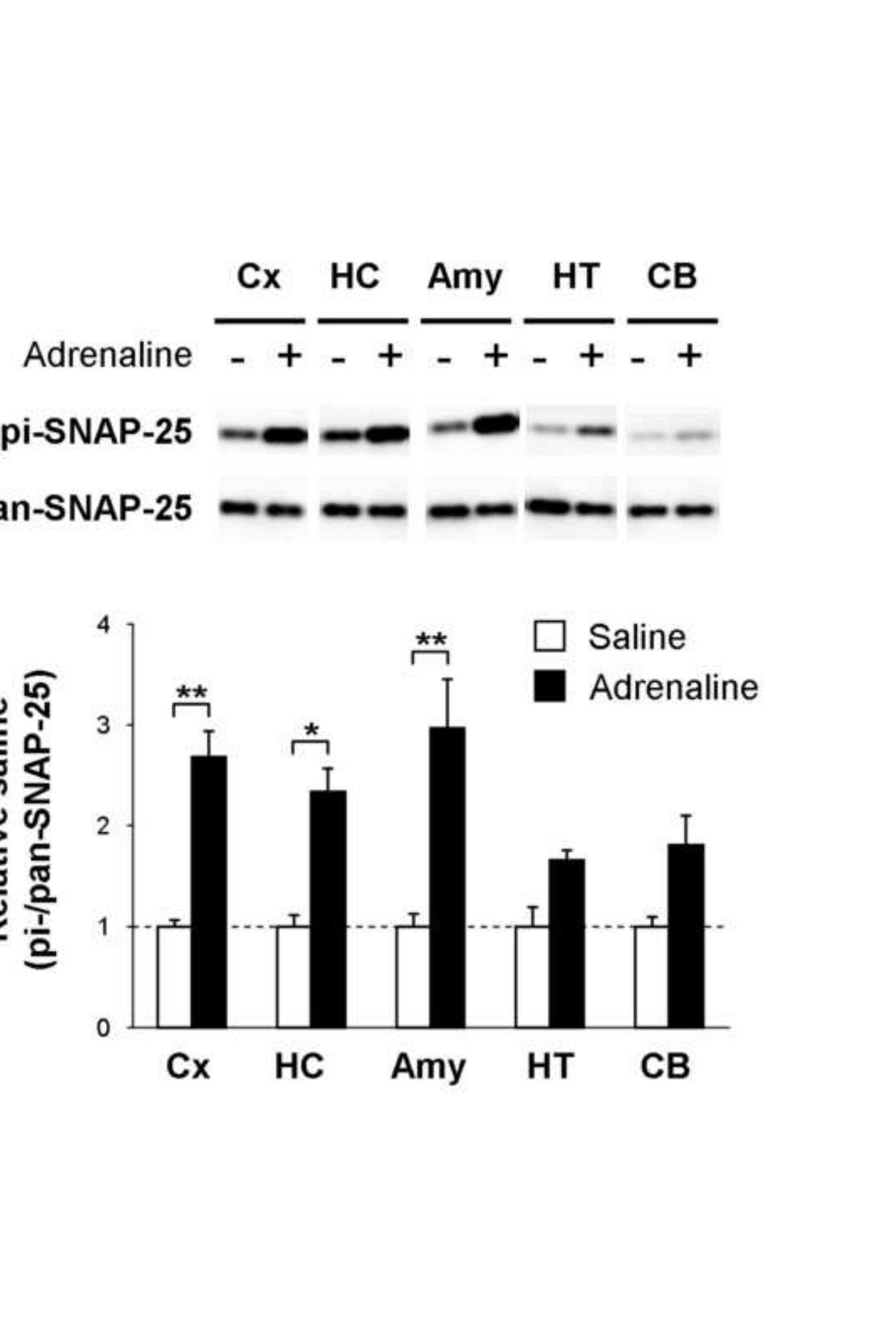




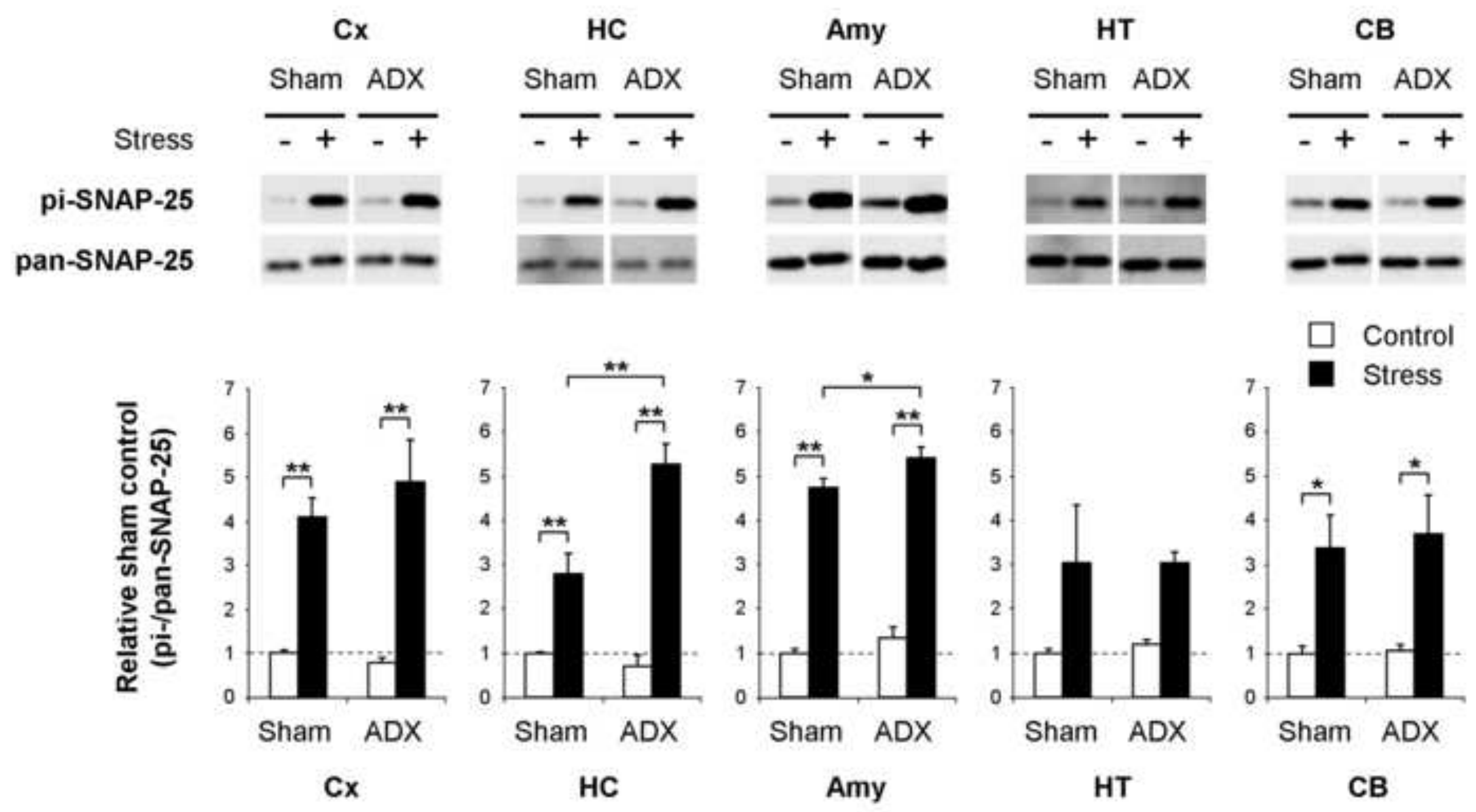

HC

Amy

HT

CB 


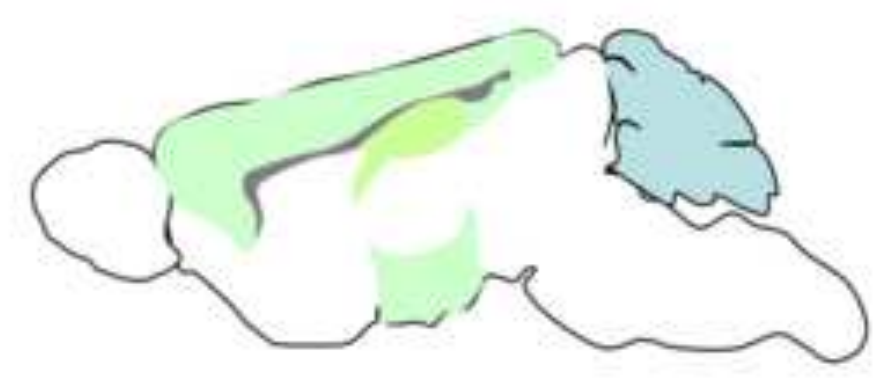

SNAP-25
dephosphorylatin

SNAP-25
dephosphorylatin

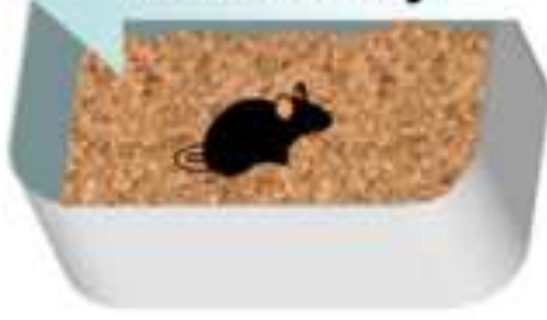

\section{Recovery}

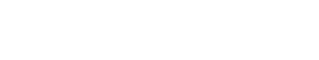

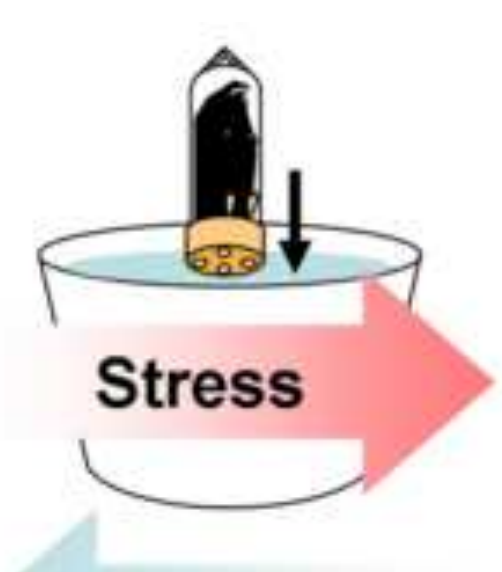

\author{
SNAP-25 \\ phosphorylatin
}

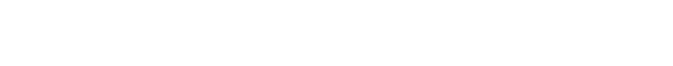

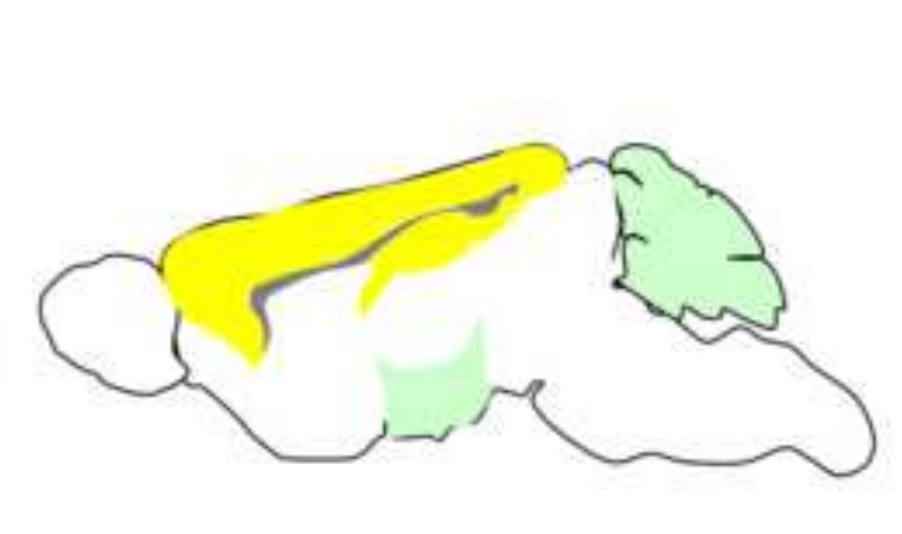

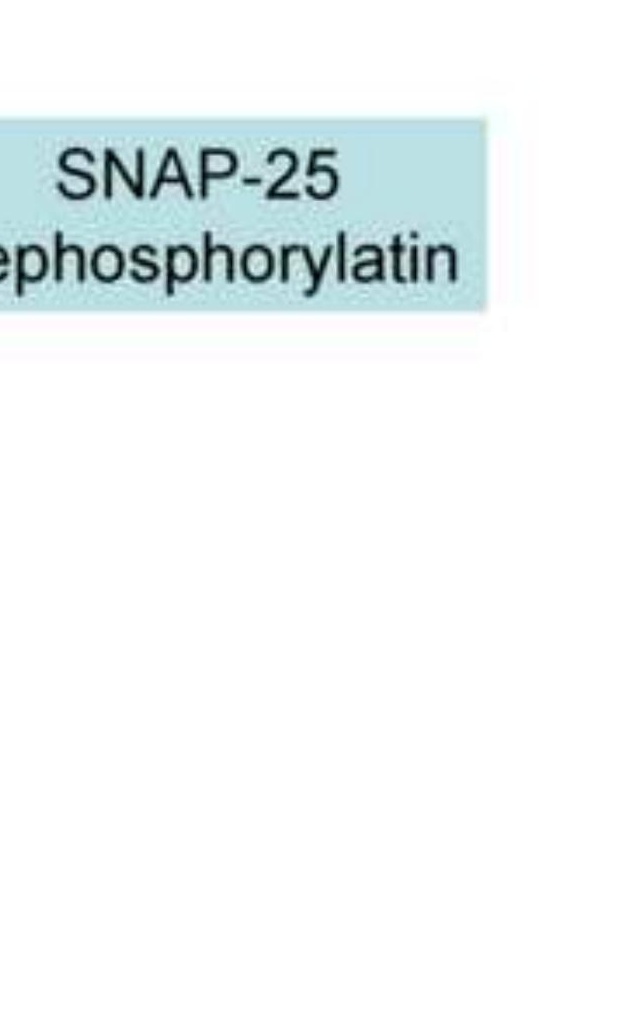

2

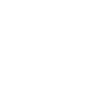

